\title{
Technology acquisition and the management of health services
}

\author{
Assessor em Desenvolvimento de Serviços de Saúde \\ OPS/OMS - Chile
}

\section{Health Services Evolution}

Probably the most remarkable characteristic of the evolution of health services in the second half of the twentieth century is the continuous and consistent increase in the costs of this type of service. The feature is constant for countries very different conditions of development and levels of health. This feature seems to be associated primarily to the maturing of the populations but also in considerable proportion, to the arrival of new technologies. A quite curious aspect of this latter phenomenon is the fact that even the arrival of technologies that theoretically should decrease costs may paradoxically increase them. An example of this phenomenon is the impact of laparoscopic cholecystectomy in the overall costs of biliary surgery. Even as the unitary cost of the procedure conducted under the new technology is considerably lower than the classic one, the huge increase in the indication of the now safer or less morbidity inducing procedure, the increase of the indication to formerly risky cases (in order of ten times, in this specific case) caused the final costs of the attention for this item, at best, to remain stacionary.

The configuration of the health services, specially in developed countries is established by the quest of cost-effectiveness. The chief feature of this new face is the extraordinary diversification of services from the classic out-patient / in patient model to a much more complex one, implicating in the development of a great variety of both ambulatory (ambulatory surgery, independent emergency unities, self care programs, utilization of alternative medicine...) and "in-patient" (home care, nursing homes, long term care, hospice care and other). The great revolution in health care seems to be, however, the shift from the patient preventive/curative model to the participative management of health risks as is the case of the "healthy communities" movement.

2. The Role of Technology in the Future of Health

\section{Services Management}

The role of technology in the present status of the health systems evolution is determined by a paradigm where it is expected that the emergent health problems, consequence of aging and the evolution of the health status of the populations, will be resolved at the end tip of the epidemiological chain by new technologies, essentially curative in character. The social value of strong preventive technologies like measles or polio immunization is considerable lower than expensive measures that deal (sometimes with appallingly low cost effectiveness) with the already manifested cases, as in the case of radiotherapy for advanced cancers. The extraordinary cost effectiveness of eradication of certain diseases is barely commented in the media as is the case of small pox eradication and more recently the interruption of transmission of polio in several countries. There are cases, like the eradication of natural occurring Chagas' disease in Sao Paulo State, some years ago, which was scarcely commented by the media - surely a marketing flaw by the health authorities.

The design of more socially relevant technologies need the institutionalization of health technology assessment and the discussion with society at large of the concept of socially relevant health technologies that may change the direction and intensity of the flux of resources bound to new technological development.

3. Managed Health Care and Managed Competition

One of the chief characteristics of the classical model of medical care is the tendency of the practitioner to offer all the possible alternatives of care (or technologies) to his or her client, with only marginal consideration of its cost effectiveness. This phenomenon could be called the maximalist ethos of medical practice and has deep historical roots (at least since the Hippocratic period). This characteristic explains, at least in part, why competent and honest professionals may counsel families to waste scarce familiar resourses and to dramatically mobilize limited social resourses to travel abroad with their severely ill relativies in the search of expensive treatments with questionable results.

Managed care has been a resourse that the health care establishment recurred to control the cost spiral in health ser- 
vices. It includes some modification in the classical model of health services delivery (free choice of the practitioner by the patient and free choice of procedures and technologies by the practitioner). The alternatives of managed care involves some limitations to the classical model. The most commom example, the HMOs in the US, in general, offer a list of phisicians for choice by the engaged population (these physicians, if not hired, charge pre-agreed fees and use ancillary resources limited in its variety, origin, or conditions of use).

Managed Competition is a concept developed recently in the health care management literature and involves mechanisms to permit that the one patient/one provider/one financier model may not degenerate into low quality services, specially from the patient (and his/her family) standpoint. Managed competition allows users to choose between a limited choice of care providers and these ones to choose among a limited choice of ancilliary procedures or more complex care support.

\section{Care}

4. Management of Technology Acquisition in Health

One of the most cherished dreams of health care managers and financiers is the possibility of somehow manage the incorporation of new technologies and procedures into the health systems they are responsible for. Certificates of Need and some limitations in the covered procedures are some of the available alternatives with this purpose. Sheer economical deficiencies are the most commom mechanism of limitation of technology incorporation in poorer countries (when we consider the cost of an installed Positron Emission Tomograph, circa US\$ 8 million, it is easy to conclude that this mechanism may not be absent even in developed countries or richer health systems).

The feeling that some type of management of technology incorporation or utilization is indispensable to the financial equilibrium of the health care systems is almost universal. The most common alternatives of regulation deal much more with the security of health equipment than with the effectiveness of their utilization. This results in two quite common situations: 1) implementation of techniques or equipment of uncertain impact in the outcomes of the health problems they are supposed to help to resolve; and 2) the change of purpose of certain equipment during the history of their utilization. An example of the first situation is the explosion of hyperbaric equipment three decades ago. Results of investigation slowly demonstrated the ineffectiveness of this type of equipment in most of its former indications. The second type of phenomenon is exemplified by the progressive change in the utilization of Computerized Tomography, proposed in the mid seventies for the assessment of cerebral tumors, this type of equipment progressively expanded its spectrum of utilization into much more common occurrences like cerebro-vascular accidents and head injuries.

The uncertainty associated with the effectiveness and adequacy of new technologies in health care rends manda- tory some type of initiative bound to control their negative impacts.

4.1. Heath Practice Standardization: Guidelines,

\section{Protocols and Critical Pathways.}

Since the invention, in the beginning of the 50's of the controlled clinical trial with random allocation of cases, this technique became progressively the standard for the evaluation of procedures in health care. As a consequence, the ultimate alternative to warrant effectivess in heath care is associated with the diagnostic and therapeutic standardization resulting from such type of trials. Under the implamentation of this type of technique, as in the case of cancer care, the distinction between investigation and everyday practice results blurred.

Protocols often deal with the complete cycle of caring for a specific diagnosis (as in the case of cancer), but there are other very interesting alternatives of protocol utilization as in the rationalization of a given procedure or equipment. The Radiology Service at the Hospital das Clínicas of the São Paulo University uses for three years, protocols to deal with the utilization of Magnetic Resonance. A coordinator for each specialty (e.g. Neurology and Neurosurgery) examines every and each prescription of the procedure and compares it with a set of pre-agreed rules that determine when the procedure is indicate for each one of the chief situations observed in the practice of that especiality.

A less rigorous alternative to the practice under protocols is the use of Guidelines. These are orientations of different level of detail, based in the practice of experts (best practices in use) and the reports of literature and its goal is to rend the practice more economical and effective, in the same way.

Critical pathways were developed to include standardization of medical, nursing, ancillary and management procedures so to obtain increases in effectiveness and quality and optimization to resource utilization in very common procedures (diabetic comma, treatment, as an example).

\section{Health Plans}

Another type of intervention, now at the level of services consumption are the basic plans (or basic baskets of services). This type of instrument, that received intensive analysis in the literature specially after its utilization in the Oregon experience and the proposal of a National Health Plar by the Clinton Administration, consist in the development of a set of pairs diagnosis/procedures through consultation of specialists, managers and clients, in general based in its desirability from the efectiveness and social value standpoint. The development of basic health plans requires, often, the utilization of a cut point in the large prioritized list of pairs, to match needs with resource availability.

\section{Final Remarks}

Technology has been viewed by the population as well as by politicians and health care professionals as a cornucopia of 
miracle solutions for the changing and engrossing list of health needs of populations at different levels of development. The mythical nature of the perception of the capabilities of most technologies (process' and product's technologies), although considerably discussed at scientific meetings and papers, never received an adequate consideration by the media, or by scientific popularization means. The shift of emphasis from clinical problem solution to community (or organization) based risk manegement, seems to be an acceptable alternative to the current situation of technology misuse.

Protocols, guidelines, critical pathways, the discussion and implementation of basic health plans, as well managed care and managed competition seem to be alternatives for the future solution for this severe problem. Healthy Communities (or organizations) may be the environment were all these solutions may be envisioned, implemented and optimized. 